\title{
The effect of $3 \%$ and $6 \%$ hypertonic saline in viral bronchiolitis: a randomised controlled trial
}

\author{
Jasmijn Teunissen ${ }^{1}$, Anne H.J. Hochs ${ }^{1}$, Anja Vaessen-Verberne ${ }^{2}$, \\ Annemie L.M. Boehmer ${ }^{3}$, Carien C.J.M. Smeets ${ }^{4}$, Hein Brackel ${ }^{5}$, \\ René van Gent ${ }^{6}$, Judith Wesseling ${ }^{7}$, Danielle Logtens-Stevens ${ }^{1}$, Ronald de Moor ${ }^{8}$, \\ Philippe P.R. Rosias ${ }^{9}$, Steph Potgieter ${ }^{10}$, Marianne R. Faber ${ }^{11}$, \\ Han J.E. Hendriks ${ }^{12}$, Maryska L.G. Janssen-Heijnen ${ }^{13}$ and Bettina F. Loza ${ }^{1}$
}

Affiliations: 'Dept of Paediatrics, VieCuri Medical Centre, Venlo, The Netherlands. ${ }^{2}$ Dept of Paediatrics, Amphia Hospital, Breda, The Netherlands. ${ }^{3}$ Dept of Paediatrics, Maasstad Hospital, Rotterdam, The Netherlands. ${ }^{4}$ Dept of Paediatrics, Elisabeth Hospital, Tilburg. The Netherlands. ${ }^{5}$ Dept of Paediatrics, Catharina Hospital Eindhoven, The Netherlands. ${ }^{6}$ Dept of Paediatrics, Máxima Medical Centre, Veldhoven, The Netherlands. ${ }^{7}$ Dept of Paediatrics, Rijnstate Hospital, Arnhem, The Netherlands. ${ }^{8}$ Dept of Paediatrics, Twee Steden Hospital, Tilburg. The Netherlands. ${ }^{9}$ Dept of Paediatrics, Orbis Medical Centre, Sittard, The Netherlands. ${ }^{10}$ Dept of Paediatrics, Laurentius Hospital, Roermond, The Netherlands. ${ }^{11}$ Dept of Paediatrics, Elkerliek Hospital, Helmond, The Netherlands. ${ }^{12}$ Dept of Paediatrics, Maastricht University Medical Centre, Maastricht, The Netherlands. ${ }^{13}$ Dept of Clinical Epidemiology, VieCuri Medical Centre, Venlo, The Netherlands.

Correspondence: Bettina F. Loza, Tegelseweg 210, 5912 BL Venlo, The Netherlands. E-mail: blozadviecuri.nl

ABSTRACT Bronchiolitis is a common disorder in young children that often results in hospitalisation. Except for a possible effect of nebulised hypertonic saline (sodium chloride), no evidence-based therapy is available. This study investigated the efficacy of nebulised 3\% and $6 \%$ hypertonic saline compared with $0.9 \%$ hypertonic saline in children hospitalised with viral bronchiolitis.

In this multicentre, double-blind, randomised, controlled trial, children hospitalised with acute viral bronchiolitis were randomised to receive either nebulised 3\%, $6 \%$ hypertonic saline or $0.9 \%$ normal saline during their entire hospital stay. Salbutamol was added to counteract possible bronchial constriction. The primary endpoint was the length of hospital stay. Secondary outcomes were need for supplemental oxygen and tube feeding.

From the 292 children included in the study (median age 3.4 months), 247 completed the study. The median length of hospital stay did not differ between the groups: $69 \mathrm{~h}$ (interquartile range 57), $70 \mathrm{~h}$ (IQR $69)$ and $53 \mathrm{~h}($ IQR 52$)$, for $3 \%(n=84)$ and $6 \%(n=83)$ hypertonic saline and $0.9 \%(n=80)$ normal saline, respectively, $(p=0.29)$. The need for supplemental oxygen or tube feeding did not differ significantly. Adverse effects were similar in the three groups.

Nebulisation with hypertonic saline (3\% or $6 \%$ sodium chloride) although safe, did not reduce the length of stay in hospital, duration of supplemental oxygen or tube feeding in children hospitalised with moderateto-severe viral bronchiolitis.

0 @ERSpublications

Hypertonic saline nebulisation did not reduce hospital stay for children with viral bronchiolitis http://ow.ly/xRVVx

For editorial comments see page 827

Received: Sept 112013 | Accepted after revision: May 162014 | First published online: June 252014

Clinical trial: The trial is registered at The Dutch Trial Register, number NTR1494 and the EU Clinical Trials Register, EudraCT, number 2008-003886-17.

Support statement: Financial support was provided for the study by educational grants from Schering Plough B.V. (Maarssen, The Netherlands), Nutricia (Zoetermeer, The Netherlands) and the Christine Bader Foundation from Irene Children's Hospital (Arnhem, The Netherlands).

Conflict of interest: Disclosures can be found alongside the online version of this article at www.erj.ersjournals.com

Copyright (CERS 2014 


\section{Introduction}

Acute viral bronchiolitis is a viral infection of the lower respiratory tract in infants $[1,2]$. Common viruses that cause bronchiolitis include respiratory syncytial virus (RSV), human metapneumovirus, adenovirus, (para)influenza virus, rhinovirus and coronavirus [3]. Bronchiolitis is the leading cause of infant hospitalisation during the first year of life and impacts millions of infants worldwide [4]. The annual costs due to hospitalisation for bronchiolitis amount to more than \$500 million in the USA alone [5]. So far, potentially therapeutic interventions, like nebulised corticosteroids, epinephrine, beta agonists, antibiotics and recombinant human deoxyribonuclease have been shown not to be effective [6-10]. Therefore, treatment of bronchiolitis remains supportive with supplemental oxygen, maintaining fluid balance and mechanical ventilation, if required [11].

Airway oedema and mucus plugging are the predominant pathological features in infants with acute viral bronchiolitis [12]. Nebulised hypertonic saline (HS), using sodium chloride, may have positive effects on bronchiolitis, as it may reduce submucosal swelling, decrease inflammatory mediators and the viscosity of mucus and improve mucociliairy clearance [13-16]. In vitro, HS improves mucus rheologic properties (elasticity and viscosity) and accelerates mucus transport rates [17]. Studies in the past decade have suggested that nebulised mildly HS reduced the duration of stay in hospital for infants with mild-tomoderately severe bronchiolitis [4, 18-25]. All but one of these studies included small numbers of patients from single centres and used poorly defined outcomes; study groups were heterogeneous and some were not clearly defined [4, 26-28]. Studies in cystic fibrosis patients have shown that nebulised HS in a concentration of $5-7 \%$ is more effective than isotonic saline, and this effect appeared to be dose dependent [29-34]. In infants aged 4 months and older with cystic fibrosis, HS was proven to be safe [35]. Therefore, we performed a randomised, controlled trial of different concentrations of nebulised HS in infants hospitalised for viral bronchiolitis. We hypothesised that nebulisation with HS would dose-dependently reduce the length of hospital stay and the duration of supportive therapy.

\section{Methods and materials}

\section{Setting}

This double-blind, randomised, controlled trial was conducted in 11 general hospitals and one tertiary medical centre in the Netherlands. The study was approved by the central medical ethics committee of the Netherlands (The Dutch trial register, number: NTR 1494) and the local ethics committees of all participating hospitals. On behalf of each child, at least one legal caregiver signed an informed consent form before study start.

\section{Patients}

Children aged 0-24 months were eligible for the study if they were admitted to one of the participating hospitals because of mild-to-severe viral bronchiolitis and had a Wang score $\geqslant 3$ at presentation. The Wang clinical severity scoring consists of four items: respiratory rate, wheezing, retractions and general condition (table 1) [36]. The diagnosis "bronchiolitis" was clinically defined by symptoms of an upper respiratory tract infection with wheezing, tachypnoea and dyspnoea [11]. To reduce the likelihood of including children with atopic wheeze, all children received a single inhalation with $2.5 \mathrm{mg}$ salbutamol before entering the study. Children were excluded if the Wang score improved at least 2 points after inhalation. Further exclusion criteria were haemodynamically important congenital heart disease, chronic pre-existent lung disease, T-cell immunodeficiency, treatment with corticosteroids, and previous wheezing, (food) allergy or eczema.

\section{Study medication}

Study medication for all centres was prepared by an internationally certified research pharmacist (Sterop Pharmacobel, Brussels, Belgium). To prevent possible bronchial obstruction, $2.5 \mathrm{mg}$ salbutamol was added to each dose. The saline concentrations were calculated so that the concentration of the definitive solution with salbutamol was $0.9 \%, 3 \%$ or $6 \%$ sodium chloride and the total volume was $4 \mathrm{~mL}$ per inhalation.

All participants, care givers and medical staff were blinded to the composition of the study solutions, which were identical in vial packaging, colour, smell and other physical characteristics. The vials were used for up to $24 \mathrm{~h}$ after opening. The expiry date of all vials was December 31, 2011. The code was deposited at the pharmacist.

\section{Study design}

The patients were recruited between November 2009 and May 2011, during the bronchiolitis season. On admission the clinical history of the patient was recorded, which included duration of symptoms prior to admission, use of medication and the patient and family history of atopy. A nasopharyngeal swab was 
TABLE 1 The Wang clinical severity scoring system for lower respiratory tract infections in infants

Wang score

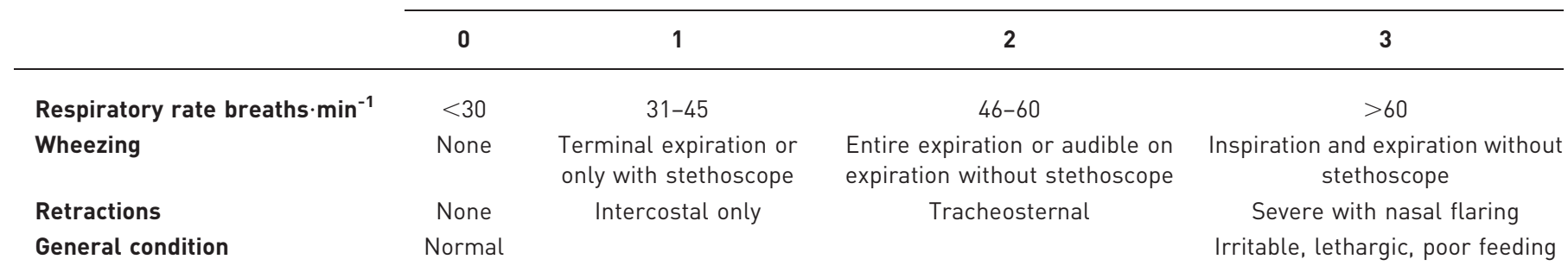

Reproduced from [36] with permission from the publisher.

obtained for viral analysis. If a child fulfilled the inclusion criteria and parental consent was obtained, nebulisation with the study medication was started within $12 \mathrm{~h}$ of admission.

All selected patients were randomly assigned to one of the two intervention groups or to the control group. Randomisation was done per centre and clustered in blocks of six patients. Each patient received a consecutively randomised number that corresponded to identical $20 \mathrm{~mL}$ vials, which contained the different sodium chloride solutions. The intervention groups received inhalations with $3 \%$ or $6 \% \mathrm{HS}$, and the control group received inhalations with $0.9 \%$ sodium chloride (normal saline (NS)).

The solutions were nebulised every $8 \mathrm{~h}$ with a constant oxygen flow of $6-8 \mathrm{~L} \cdot \mathrm{min}^{-1}$ from a wall outlet in combination with a HOT Top Plus Nebuliser (Intersurgical, Uden, The Netherlands), Mass Median Aerodynamic Diameter (MMAD) $4 \mu \mathrm{m}$, via a tight-fitting face mask and were administered until empty. Nebulisations were continued until discharge.

All children were evaluated twice a day, which was based on physical examination, the Wang score, heart rate, saturation, respiratory rate, need for supplemental oxygen and tube feeding. The evaluations were done by the paediatrician on duty. Before the start of the study all participating medical staff were trained how to evaluate the patients and classify the Wang clinical severity scoring system in order to improve interobserver agreement.

According to predefined criteria, supplemental oxygen was started in infants with a room air saturation of $93 \%$, or lower, during $>10$ min or acute deoxidisation of $<85 \%$. This was stopped when saturation was consistently $>93 \%$. The indication for starting and stopping tube feeding was a minimal intake calculated as $75 \%$ of normal intake. Fluid loss because of dehydration or diarrhoea was compensated by the addition of lost fluid to the minimal intake. Additional medication and other supportive care were given according to hospital guidelines.

All additional medication, time and quantity of supplemental oxygen and tube feeding were recorded in the case record form, as were heart rate, oxygen saturation, temperature and adverse events. Adverse reactions were defined as any undesirable effect observed, irrespective of whether it was considered related to the medication being investigated or not. Possible known side-effects of inhalation with HS included rhinorrhea, restlessness, coughing and bronchial obstruction $[4,34,37]$.

The primary outcome was duration of hospital stay, which was calculated as the number of hours between the first dose of study medication and the clinical decision to discharge. In this way, the primary endpoint was not influenced by additional hospital stay due to social or administrative factors. Protocol-defined discharge criteria included no need for supplemental oxygen, tube-feeding or intravenous fluids, according to the responsible paediatrician. Secondary outcomes were transfer to a Paediatric Intensive Care Unit (PICU) because of respiratory insufficiency, need and duration of supplemental oxygen or tube feeding. The safety of the treatments was measured by registration of adverse events.

\section{Statistical methods}

Previous studies showed a decrease in the length of stay in hospital from 4 days to 3 days, after the inhalation of $3 \%$ HS [18]. Based on this $25 \%$ reduction of hospital stay, a sample size of 65 patients per trial arm was required for the current study to achieve a power of $90 \%$ with a p-value $<0.05$. All data were anonymously recorded, entered into an Excel spreadsheet (Microsoft Corp, Redmond, WA) and imported into SPSS version 19.0 software (SPSS Inc, Chicago, IL) for analysis. Analyses were done according to the intention-to-treat and per protocol principles. Differences between included and excluded patients, with 
respect to patient characteristics, were evaluated by means of independent t-test (age) and Chi-squared test (sex and intervention). All continuous variables (duration of hospital stay, hours of supplemental oxygen and tube feeding) were tested for normality. In case of normal distribution, differences between the three groups were tested with ANOVA and in case of not-normal distribution, the Kruskal-Wallis test was used. For categorical variables differences in distribution between intervention groups were tested with the Chisquared test. Univariate differences in time until hospital discharge between intervention groups were tested with the Log-rank test.

\section{Results}

Between November 2009 and May 2011, 292 previously healthy children with moderate-to-severe viral bronchiolitis were included in the study and randomised (fig 1). Prior to enrolment, most patients received nasal-decongestants: 21 received salbutamol (by spacer), 10 paracetamol, four amoxicillin, three omeprazol, three nystatine, and one received valproinic acid.

Two patients were discharged due to rapid clinical amelioration and were, therefore, withdrawn from the study before the first dose of the trial medication was given. A total of 290 infants (median age 3.4 months, range 10 days to 23 months) were enrolled in the study and analysed in the intention-to-treat analysis. 43 (15\%) patients were excluded for the per-protocol analysis and $18(6.2 \%)$ patients did not complete the treatment because of adverse events. Of these, nine patients left due to coughing or agitation during and after nebulisation and nine others because of clinical deterioration, such as increased need for oxygen, effort of breathing or progressive airway obstruction. Withdrawal of informed consent led to the withdrawal of a further nine $(3.1 \%)$ patients. Thirteen infants were excluded from the study due to protocol deviation. Three patients (1.2\%) required mechanical ventilation at a PICU during the course of disease: one patient deteriorated $5 \mathrm{~h}$ after the first inhalation with the $6 \% \mathrm{HS}$; the clinical condition of one patient, randomised to the $6 \%$ group, worsened after 6 days of treatment and required intensive care admission; and one patient, receiving NS, had been respiratory insufficient on admission and this clinical condition did not change after

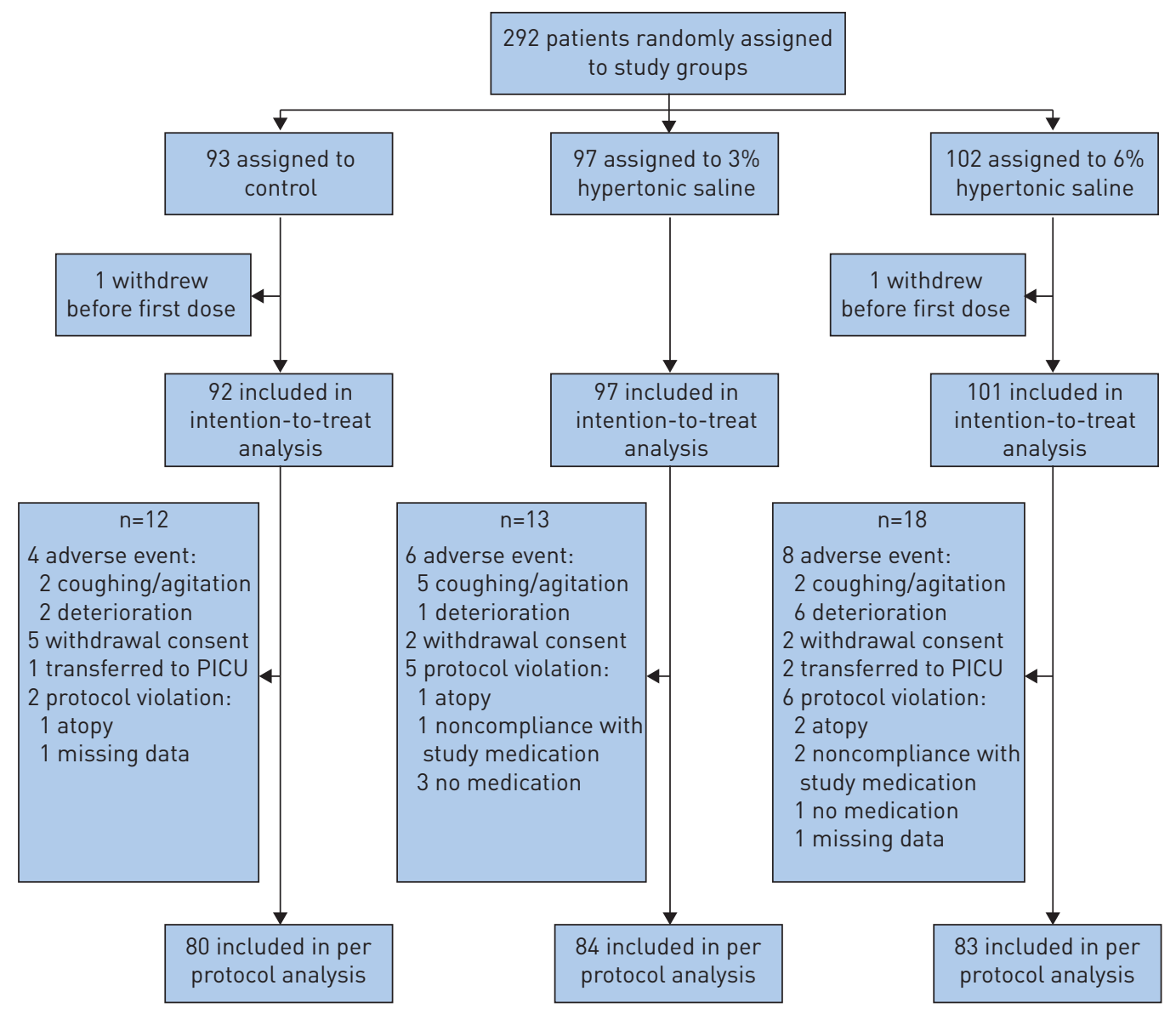

FIGURE 1 Enrolment of patients to the study and the follow-up and analyses procedures. 
receiving one dose of the study medication while awaiting transfer to the PICU (fig. 1). The number of withdrawals (per reason) did not significantly differ between intervention groups $(p=0.47)$.

In total, 247 infants completed the study and were analysed in the per-protocol analysis; 84 received 3\% HS, 83 received 6\% HS and 80 received NS (fig. 1). Patient baseline characteristics did not significantly differ between the three groups (table 2). Results of the intention-to-treat and per-protocol analyses did not differ for baseline characteristics or for any primary or secondary outcomes (data not shown). Besides the study medication, patients received: nasal-decongestants $n=161$, paracetamol $n=30$, antibiotics $n=11$, salbutamol $n=6$, ibuprophen $n=1$, nystatine $n=1$, and ranitidine $n=1$. The use of nasal decongestant (xylomethazoline and sodium chloride) was equally distributed among the study groups (data not shown).

The median duration of hospital stay was $69 \mathrm{~h}$ in the 3\% HS group, $70 \mathrm{~h}$ in the $6 \%$ HS group and $53 \mathrm{~h}$ in the NS group $(p=0.29)$ (table 3). Differences in time to discharge between the three groups were not statistically significant ( $\mathrm{p}=0.26)$ (fig. 2).

Supplemental oxygen was needed in 50 (59.5\%) infants in the 3\% HS group, 53 (63.9\%) infants in the 6\% HS group, and $51(63.8 \%)$ infants in the $0.9 \%$ NS group $(\mathrm{p}=0.70)$, with a median duration of 54,54 and 40 h, respectively, $(\mathrm{p}=0.14)$.

Additional tube feeding was required by 29 (34.5\%) infants in the 3\% HS group, 31 (37.3\%) infants in the $6 \%$ HS group and $22(27.5 \%)$ infants in the $0.9 \%$ NS group $(p=0.39)$, with a median duration of 62,52 and $54 \mathrm{~h}$, respectively, $(\mathrm{p}=0.87)$ (table 3$)$.

The median Wang score at discharge was 2.0 in the NS group and in the 3\% HS group and 1.0 in the $6 \%$ HS group $(\mathrm{p}=0.53)$. The Wang score at discharge improved regardless of the treatment regimens (tables 3 and 4 ), without significant differences between the groups $(\mathrm{p}=0.80)$.

A substantial number of adverse effects were noted in all treatment groups (table 5). Except for cough, which occurred significantly more often in the HS groups $(p=0.03)$, no differences were found between groups. Withdrawals because of adverse events did not differ between groups $(p=0.59)$. Most adverse events were likely to be related to the underlying viral bronchiolitis.

\section{Discussion}

In this double-blind, randomised, multicentre trial we compared $3 \%$ and $6 \%$ HS with NS in children hospitalised with viral bronchiolitis. Neither the 3\% HS nor the 6\% HS reduced the duration of stay in hospital, the Wang clinical severity score at discharge, the duration of supplemental oxygen or tube feeding compared to NS. These results are in contrast with results published in the literature [18-22]. Pooled results from three small studies [18-20] demonstrated a reduction in the duration of hospitalisation by 0.9 days in favour of the 3\% HS group when compared with the NS group, whereas LUO and colleagues [21, 22] found a reduction by 1.4 and 1.6 days, respectively, in their studies in mild-to-moderate and moderate-to-severe bronchiolitis. However, AL-ANSARI et al. [24] compared nebulisation of 3\% HS and 5\% HS to NS in relatively mild bronchiolitis in infants staying at a short-stay unit and did not show an effect on duration of stay at hospital, which he described as affected by medical and social factors.

Although criteria for diagnosis of viral bronchiolitis were less well defined in most of these studies except in the study of KUZIK et al. [20], differences in study populations between our study and previous studies are unlikely to explain the differences between the different results. Our study population was comparable with

TABLE 2 Baseline characteristics of enrolled infants

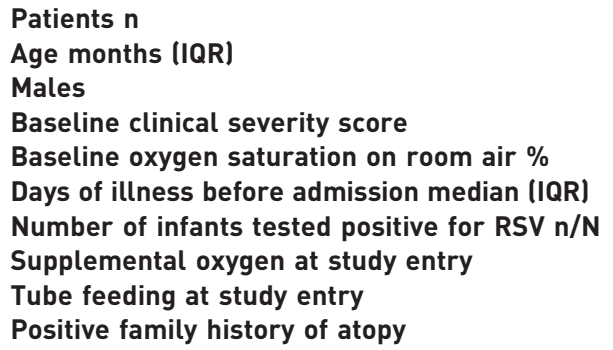

\section{4}

$3.6(5.2)$

$44(52.4)$

$6.2 \pm 1.9$

$95.4 \pm 3.6$

$4.0(3.0)$

$67 / 80$

$34(40.5)$

$18(21.4)$

33 (39)

\section{3}

$3.4(3.8)$

$48(57.8)$

$6.2 \pm 2.2$

$95.4 \pm 3.4$

4.0 (3.0)

$75 / 82$

$36(43.4)$

$20(24.1)$

$31(37)$
$6.2 \pm 2.1$

$95.5 \pm 3.4$

4.0 (3.5)

$70 / 79$

$36(45)$

$13(16.3)$

$29(36)$

Data are presented as $\mathrm{n}(\%)$ or mean $\pm \mathrm{SD}$, unless otherwise stated. IQR: interquartile range; RSV: respiratory syncytial virus. 


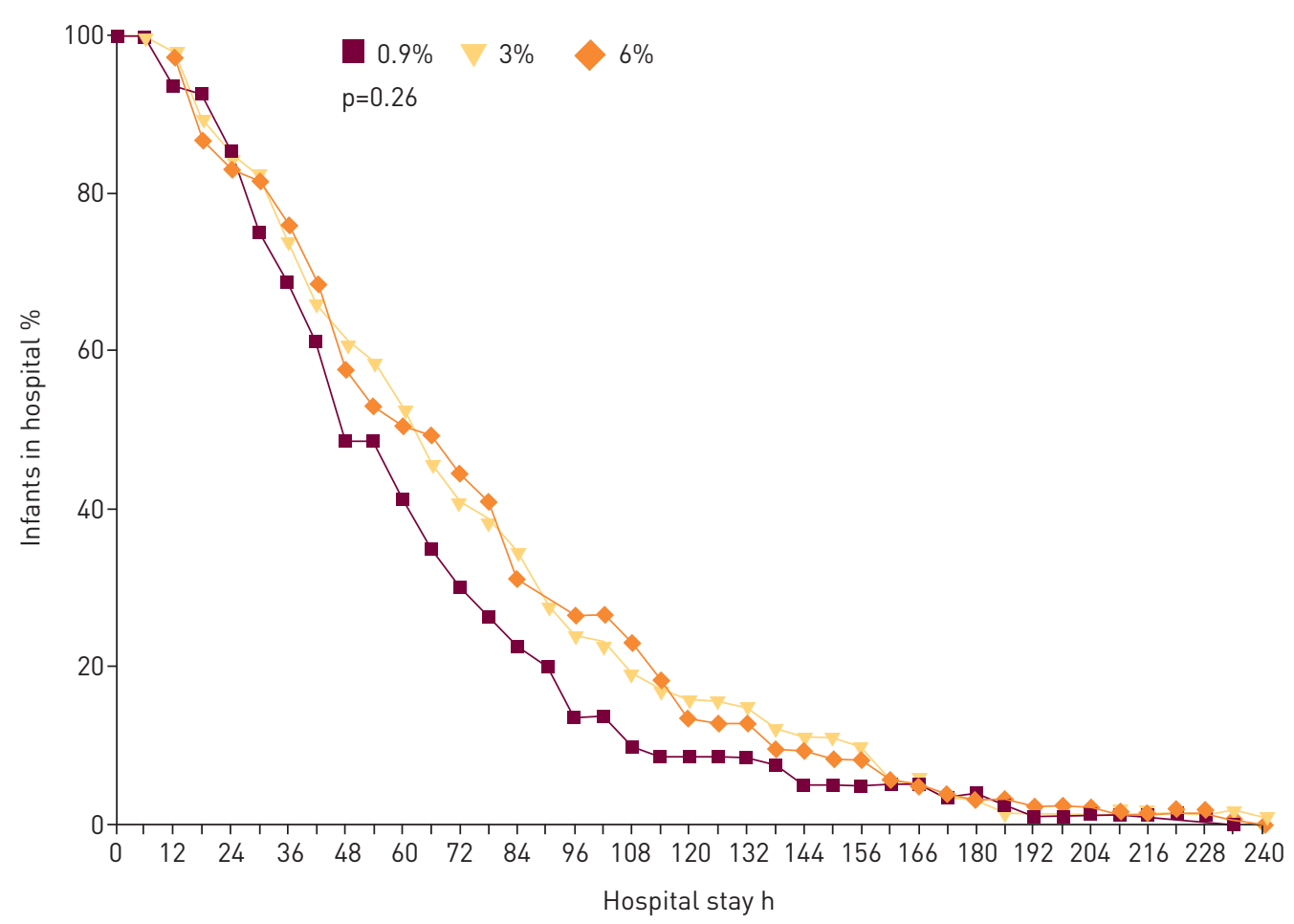

FIGURE 2 The percentage of infants remaining in hospital displayed against time until discharge, according to intervention.

respect to age, clinical severity, duration of illness before admission and predominantly RSV-positive infants. Additionally to the exclusion criterion of previous wheezing, atopic manifestations, such as eczema or food allergy, we tried to avoid inclusion of infants who might benefit from salbutamol. Indeed, a recent study showed that in children with bronchiolitis, the Wang score improved significantly more in atopic children than in nonatopic children when salbutamol was added to the medication [23], which supports our approach. Taking into account the possible rapid responders to a single dose of salbutamol [12], it could be argued that our study protocol led to the exclusion of rapid responders, possibly influencing the results. In total, $14 \%$ of all children screened for inclusion had to be excluded because of a positive reaction to salbutamol. However, even if these infants had been included and randomly assigned to the study groups, the number would have been too small to influence the results significantly.

A meta-analysis suggested an inverse relationship between severity of disease and effect of nebulised HS [4]. Hence, differences in severity of bronchiolitis between the studied populations could explain the different findings. However, this does not seem to have been the case, as at baseline the Wang scores in our study were 6.2 compared to 5.8 in the study accounting for half the weight of the meta-analysis [21], while the other studies of the meta analysis included patients with higher Wang scores [18-20]. Our study was not

TABLE 3 Primary and secondary outcomes for the three intervention groups

\begin{tabular}{|c|c|c|c|c|}
\hline & $3 \%$ hypertonic saline & $6 \%$ hypertonic saline & $0.9 \%$ normal saline & p-value \\
\hline Patients $\mathrm{n}$ & 84 & 83 & 80 & \\
\hline Length of hospital stay $\mathrm{h}$ median (IQR) & $69(57)$ & 70 (69) & $53(52)$ & 0.29 \\
\hline \multicolumn{5}{|l|}{ Supplemental Oxygen } \\
\hline Patients n $(\%)$ & $50(60)$ & $53(64)$ & $51(64)$ & 0.81 \\
\hline Duration ${ }^{\#} \mathrm{~h}$ median (IQR) & $54(48)$ & $54(61)$ & $40(41)$ & 0.14 \\
\hline \multicolumn{5}{|l|}{ Tube feeding } \\
\hline Patients n $(\%)$ & $29(35)$ & $31(37)$ & $22(28)$ & 0.39 \\
\hline Duration ${ }^{\#} \mathrm{~h}$ median (IQR) & $62(58)$ & $52(55)$ & $54(83)$ & 0.87 \\
\hline Median Wang score at discharge & 2.0 & 1.0 & 2.0 & 0.53 \\
\hline Mean improvement of Wang score at discharge & -4.55 & -4.54 & -4.33 & 0.80 \\
\hline
\end{tabular}

\footnotetext{
\#: patients without oxygen, respectively tube fed, were excluded for analyses; IQR: interquartile range.
} 
TABLE 4 Median Wang score after 24,48 and $72 \mathrm{~h}$ for the three intervention groups

$3 \%$ hypertonic saline

$6 \%$ hypertonic saline

$0.9 \%$ normal saline

$\begin{array}{lccc}\text { Patients n } & 84 & 83 & 80 \\ \mathbf{2 4} \text { h } & & & 75(94) \\ \quad \text { Patients n (\%) } & 80(95) & 78(94) & 4.0(4) \\ \quad \text { Wang score median (IQR) } & 4.0(3) & 4.0(3) & 60(75) \\ \mathbf{4 8} \mathbf{~} & & 62(75) & 3.0(3) \\ \quad \text { Patients n (\%) } & 66(79) & 3.0(3) & 35(44) \\ \quad \text { Wang score median (IQR) } & 3.0(3) & 41(49) & 3.0(4) \\ \mathbf{7 2} \text { h } & 42(50) & 4.0(4) & \\ \quad \text { Patients n (\%) } & 3.0(3) & \end{array}$

IQR: interquartile range.

designed to examine subgroups with different disease severity, and further studies should elucidate if HS might be specifically useful in subgroups of infants with more severe disease.

We cannot rule out that differences in type of nebulisers and frequency of nebulisation played a role. We used the same nebuliser with a MMAD of $4 \mu \mathrm{m}$ in all study patients, leading to a deposition mainly in the lower airways [38]. Potentially, a nebuliser with smaller MMAD and another lung deposition might be of influence [39]. The type of nebuliser and MMAD were not described in the other studies, therefore, subsequent comparison is not possible. Only two previous studies contained participants who had been nebulised more frequently than in our study $[20,22]$. However, there was no kinetic data on the half-life of the effect of HS and frequent nebulisation is not advised [20]. Hence, it is unlikely that differences in type of nebulisation and frequency of nebulisation could explain our findings.

Most previously published studies were single centre studies. It is possible that differences like local habits of diagnosis and treatment may have influenced the results. The current study was a multicentre study, which could possibly reduce these factors. Randomisation was undertaken for each centre and, therefore, allowed an analysis per centre; but we found no significant differences between the centres.

The strengths of our study include the use of strict inclusion and discharge criteria. We used a strict, clinically relevant definition for time of discharge, which was not influenced by social or administrative factors [24]. Hence, we confined the length of stay in hospital to the time in hospital due to medical need. This might explain our relatively short durations for hospital stay (53, 69 and 70 h for NS, 3\% HS and 6\% HS, respectively), compared with 2.6-6.0 days for 3\% HS and 3.0-7.4 days for NS in previous studies [18-22]. None of these studies took care of potentially influencing social and administrative factors; most studies only used physician-based clinical decisions.

A possible explanation why this study did not find a difference between the intervention groups and the control group could be that NS may not be a true placebo. However, the design of our study cannot rule out a positive effect of NS nebulisation in infants with viral bronchiolitis. This question could be addressed by a noninferiority trial comparing NS and HS. Physiologically, an adequate quantity of airway lining fluid is crucial for normal functioning of airway mucociliary clearance. Bronchiolitis is known to deplete water content of the airway lining fluid, causing a reduction of mucociliary clearance [12]. In vivo, the change in airway surface liquid and the improvement in mucus clearance after NS or HS inhalation is probably a direct effect of the total mass of sodium chloride added to the airway surface [13]. In ambulatory bronchiolitis patients, nebulisation with a high volume of NS was as effective as that of a lower volume of $3 \%$ HS [40]. So far, this has not been shown for more severe illnesses. Earlier studies that showed a positive effect of $3 \%$ HS also performed nebulisation three times a day, which resulted in the same total dose $[18,19,21]$. Consequently, although a higher dose of total sodium chloride may have influence, it is unlikely to explain the different study results. Our findings were supported by a recent study comparing NS to 7\% HS and using an inhalation frequency of four times a day that did not show a positive effect of HS, even with a higher total dose of sodium chloride [41]. IPEK et al. [23] compared the effect of nebulised NS and HS with and without salbutamol in outpatients with mild bronchiolitis. All study groups showed a significant decrease in clinical severity score, without differences between the groups, suggesting that NS might have some therapeutic effect as well in non-atopic patients. This supports our findings that no significant differences could be found between the use of HS and NS in bronchiolitis patients. 
TABLE 5 Adverse events recorded in the enrolled infants

$3 \%$ hypertonic saline

$\begin{array}{cl} & 84 \\ 20 & (23.8) \\ 2 & (2.4) \\ 33 & (39.3) \\ 27 & (32.1) \\ 0 & (0) \\ 11 & (13.1) \\ 1 & (1.2) \\ 1 & (1.2) \\ 0(0) & 95 \\ 4 & (4.8)\end{array}$

$6 \%$ hypertonic saline $\quad 0.9 \%$ normal saline

p-value

Patients
Coughing
Bronchial obstruction
Agitation
Rhinorrhea
Dry mucosae
Resistance
Vomiting
Saturation dips
Tachycardia $>200 \cdot \mathrm{min}^{-1}$
Total adverse effects
Withdrawal because of adverse effects

83
$24(28.9)$
$5(6.0)$
$37(44.6)$
$27(32.5)$
$0(0)$
$20(24.1)$
$1(1.2)$
$2(2.4)$
$0(0)$
119
$6(7.2)$

$\begin{array}{cl} & 80 \\ 10 & (12.5) \\ 3 & (3.8) \\ 23 & (28.8) \\ 26 & (32.5) \\ 1 & (1.3) \\ 11 & (13.8) \\ 1 & (1.3) \\ 1 & (1.3) \\ 1 & (1.3) \\ & 76 \\ 8 & (10.0)\end{array}$

Data are presented as $\mathrm{n}$ or $\mathrm{n}(\%)$ unless otherwise stated. ${ }^{\#:}$ no Chi-squared test was performed because of the small numbers. Bold text means statistically significant.

Although no serious adverse effects were observed in the current study, a considerable number of children showed adverse effects (table 5), which largely may have been due to bronchiolitis itself. In $6.2 \%$ of all included patients, adverse effects were the reason for withdrawal. Moreover, coughing was more prevalent in both HS groups. This requires further attention, since previous studies did not include exact descriptions of adverse effects.

In conclusion, we showed that nebulised $3 \%$ HS or $6 \%$ HS did not reduce the duration of hospital stay, the Wang clinical severity score at discharge, the need for supplemental oxygen or the need for tube feeding in children aged up to 2 years who are hospitalised with viral bronchiolitis, when compared with the use of NS. Our results do not support the routine use of HS in infants with bronchiolitis.

\section{Acknowledgements}

The authors would like to thank the following who contributed towards the study: Nico Oldenhof (VieCuri Medical Centre, Venlo, The Netherlands) for the randomisation procedure; Greta Veldhuijs (VieCuri Medical Centre, Venlo, The Netherlands) for coordinating employee pharmacy; Bart Rottier (University Medical Centre, Groningen, The Netherlands) for interim analysis; Twan Mulder (University Medical Centre, Maastricht, The Netherlands) and Brigitte van der BruggenBogaarts (VieCuri Medical Centre, Venlo, The Netherlands) who were the independent doctors for patients with questions; Johan de Jongste (University medical Centre, Rotterdam, The Netherlands) for a critical review of the manuscript; and Loes Janssen (VieCuri Centre, Venlo, The Netherlands) for the control of statistical calculations.

The participating centres were VieCuri Medical Centre, Venlo; Maastricht University Medical Centre, Maastricht; Orbis Medical Centre, Sittard; Catharina Hospital, Eindhoven; Máxima Medical Centre, Veldhoven; Laurentius Hospital, Roermond; Elkerliek Hospital, Helmond; Rijnstate Hospital, Arnhem; Maasstad Hospital, Rotterdam; Amphia Hospital, Breda; Twee Steden Hospital, Tilburg; and St. Elisabeth Hospital, Tilburg, The Netherlands.

\section{References}

1 Glezen WP, Taber LH, Frank AL, et al. Risk of primary infection and reinfection with respiratory syncytial virus. Am J Dis Child 1986; 140: 543-546.

Hall CB. Respiratory syncytial virus and parainfluenza virus. N Engl J Med 2001; 344: 1917-1928.

3 Calvo C, Pozo F, García-García ML, et al. Detection of new respiratory viruses in hospitalized infants with bronchiolitis: a three-year prospective study. Acta Paediatr 2010; 99: 883-887.

4 Zhang L, Mendoza-Sassi RA, Wainwright C, et al. Nebulized hypertonic saline solution for acute bronchiolitis in infants. Cochrane Database Syst Rev 2008; 4: CD006458.

5 Pelletier AJ, Mansbach JM, Camargo CA Jr. Direct medical costs of bronchiolitis hospitalizations in the United States. Pediatrics 2006; 118: 2418-2423.

6 Fernandes RM, Bialy LM, Vandermeer B, et al. Glucocorticoids for acute viral bronchiolitis in infants and young children. Cochrane Database Syst Rev 2010: 10; CD004878.

Gadomski AM, Brower M. Bronchodilators for bronchiolitis. Cochrane Database Syst Rev 2010; 12: CD001266.

8 Hartling L, Bialy LM, Vandermeer B, et al. Epinephrine for bronchiolitis. Cochrane Database Syst Rev 2011; 6: CD003123.

9 Boogaard R, Hulsmann AR, van Veen L, et al. Recombinant human deoxyribonuclease in infants with respiratory syncytial virus bronchiolitis. Chest 2007; 131: 788-795.

10 Spurling GK, Fonseka K, Doust J, et al. Antibiotics for bronchiolitis in children. Cochrane Database Syst Rev 2007; 1 : CD005189.

11 Diagnosis and management of bronchiolitis. Pediatrics 2006; 118: 1774-1793.

12 Mandelberg A, Amirav I. Hypertonic saline or high volume normal saline for viral bronchiolitis: mechanisms and rationale. Pediatr Pulmonol 2010; 45: 36-40. 
13 Sood N, Bennett WD, Zeman K, et al. Increasing concentration of inhaled saline with or without amiloride: effect on mucociliary clearance in normal subjects. Am J Respir Crit Care Med 2003; 167: 158-163.

14 Tomooka LT, Murphy C, Davidson TM. Clinical study and literature review of nasal irrigation. Laryngoscope 2000; 110: 1189-1193.

15 King $\mathrm{M}$, et al. Rheology of cystic fibrosis sputum after in vitro treatment with hypertonic saline alone and in combination with recombinant human deoxyribonuclease I. Am J Respir Crit Care Med 1997; 156: 173-177.

16 Pavia D, Thomson ML, Clarke SW. Enhanced clearance of secretions from the human lung after the administration of hypertonic saline aerosol. Am Rev Respir Dis 1978; 117: 199-203.

17 Wills PJ, Hall RL, Chan W, et al. Sodium chloride increases the ciliary transportability of cystic fibrosis and bronchiectasis sputum on the mucus-depleted bovine trachea. J Clin Invest 1997; 99: 9-13.

18 Mandelberg A, Tal G, Witzling M, et al. Nebulized 3\% hypertonic saline solution treatment in hospitalized infants with viral bronchiolitis. Chest 2003; 123: 481-487.

19 Tal G, Cesar K, Oron A, et al. Hypertonic saline/epinephrine treatment in hospitalized infants with viral bronchiolitis reduces hospitalization stay: 2 years experience. Isr Med Assoc J 2006; 8: 169-173.

20 Kuzik BA, Al-Qadhi SA, Kent S, et al. Nebulized hypertonic saline in the treatment of viral bronchiolitis in infants. J Pediatr 2007; 151, 266-270: 270.e1.

21 Luo Z, Liu E, Luo J, et al. Nebulized hypertonic saline/salbutamol solution treatment in hospitalized children with mild to moderate bronchiolitis. Pediatr Int 2010; 52: 199-202.

22 Luo Z, Fu Z, Liu E, et al. Nebulized hypertonic saline treatment in hospitalized children with moderate to severe viral bronchiolitis. Clin Microbiol Infect 2011; 17: 1829-1833.

23 Ipek IO, Yalcin EU, Sezer RG, et al. The efficacy of nebulized salbutamol, hypertonic saline and salbutamol/ hypertonic saline combination in moderate bronchiolitis. Pulm Pharmacol Ther 2011; 24: 633-637.

24 Al-Ansari K, Sakran M, Davidson BL, et al. Nebulized 5\% or 3\% hypertonic or $0.9 \%$ saline for treating acute bronchiolitis in infants. J Pediatr 2010; 157: 630-634.e1.

25 Kuzik BA, Flavin MP, Kent S, et al. Effect of inhaled hypertonic saline on hospital admission rate in children with viral bronchiolitis: a randomized trial. CJEM 2010; 12: 477-484.

26 Villanueva P, Standish J, Douglas K, et al. Efficacy of hypertonic nebulized saline in bronchiolitis: improved outcome measures needed. J Pediatr 2011; 159: 353.

27 Morawetz D, Cheah E, Barton R, et al. Is nebulised hypertonic saline useful as an adjunctive treatment for acute bronchiolitis in infants and children less than 24 months of age? J Paediatr Child Health 2011; 47: 922-926.

28 Eber E. Treatment of acute viral bronchiolitis. Open Microbiol 2011; 5: p. 159-164.

29 Elkins MR, Robinson M, Rose BR, et al. A controlled trial of long-term inhaled hypertonic saline in patients with cystic fibrosis. N Engl J Med 2006; 354: 229-240.

30 Eng PA, Morton J, Douglass JA, et al. Short-term efficacy of ultrasonically nebulized hypertonic saline in cystic fibrosis. Pediatr Pulmonol 1996; 21: 77-83.

31 Robinson M, Daviskas E, Eberl S, et al. The effect of inhaled mannitol on bronchial mucus clearance in cystic fibrosis patients: a pilot study. Eur Respir J 1999; 14: 678-685.

32 Robinson M, Hemming AL, Regnis JA, et al. Effect of increasing doses of hypertonic saline on mucociliary clearance in patients with cystic fibrosis. Thorax 1997; 52: 900-903.

33 Subbarao P, Balkovec S, Solomon M, et al. Pilot study of safety and tolerability of inhaled hypertonic saline in infants with cystic fibrosis. Pediatr Pulmonol 2007; 42: 471-476.

34 Wark P, McDonald VM. Nebulised hypertonic saline for cystic fibrosis. Cochrane Database Syst Rev 2009; 2: CD001506.

35 Rosenfeld M, Ratjen F, Brumback L, et al. Inhaled hypertonic saline in infants and children younger than 6 years with cystic fibrosis: the ISIS randomized controlled trial. JAMA 2012; 307: 2269-2277.

36 Wang EE, Milner RA, Navas L, et al. Observer agreement for respiratory signs and oximetry in infants hospitalized with lower respiratory infections. Am Rev Respir Dis 1992; 145: 106-109.

37 Ralston S, Hill V, Martinez M. Nebulized hypertonic saline without adjunctive bronchodilators for children with bronchiolitis. Pediatrics 2010; 126: e520-e525.

38 Rubin BK, Fink JB. Aerosol therapy for children. Respir Care Clin N Am 2001; 7, 175-213: v.

39 Amirav I, Balanov I, Gorenberg $\mathrm{M}$, et al. $\beta$-agonist aerosol distribution in respiratory syncytial virus bronchiolitis in infants. J Nucl Med 2002; 43: 487-491.

40 Anil AB, Anil M, Saglam AB, et al. High volume normal saline alone is as effective as nebulized salbutamol-normal saline, epinephrine-normal saline, and 3\% saline in mild bronchiolitis. Pediatr Pulmonol 2010; 45: 41-47.

41 Jacobs JD, Foster M, Wan J, et al. 7\% hypertonic saline in acute bronchiolitis: a randomized controlled trial. Pediatrics 2014; 133: e8-e13. 